\title{
A type 2 biomarker separates relapsing-remitting from secondary progressive multiple sclerosis
}

Alex M. Dickens, PhD* James R. Larkin, PhD* Julian L. Griffin, PhD Ana Cavey

Lucy Matthews, MRCP Martin R. Turner, PhD Gordon K. Wilcock, DM Benjamin G. Davis, PhD Timothy D.W. Claridge, $\mathrm{PhD}$

Jacqueline Palace, DM

Daniel C. Anthony, PhD

Nicola R. Sibson, PhD

Correspondence to

Dr. Anthony:

Daniel.Anthony@pharm.ox.ac.uk

Editorial, page 1488

Supplemental data at Neurology.org

\section{ABSTRACT}

Objective: We tested whether it is possible to differentiate relapsing-remitting (RR) from secondary progressive (SP) disease stages in patients with multiple sclerosis (MS) using a combination of nuclear magnetic resonance (NMR) metabolomics and partial least squares discriminant analysis (PLS-DA) of biofluids, which makes no assumptions on the underlying mechanisms of disease.

Methods: Serum samples were obtained from patients with primary progressive MS (PPMS), SPMS, and RRMS; patients with other neurodegenerative conditions; and age-matched controls. Samples were analyzed by NMR and PLS-DA models were derived to separate disease groups.

Results: The PLS-DA models for serum samples from patients with MS enabled reliable differentiation between RRMS and SPMS. This approach also identified significant differences between the metabolite profiles of each of the MS groups (PP, SP, and RR) and the healthy controls, as well as predicting disease group membership with high specificity and sensitivity.

Conclusions: NMR metabolomics analysis of serum is a sensitive and robust method for differentiating between different stages of MS, yielding diagnostic markers without a priori knowledge of disease pathogenesis. Critically, this study identified and validated a type II biomarker for the RR to SP transition in patients with MS. This approach may be of considerable benefit in categorizing patients for treatment and as an outcome measure in future clinical trials.

Classification of evidence: This study provides Class II evidence that serum metabolite profiles accurately distinguish patients with different subtypes and stages of MS. Neurology ${ }^{\circledR}$ 2014;83:1492-1499

\section{GLOSSARY}

$\mathbf{A D}=$ Alzheimer disease; $\mathbf{A L S}=$ amyotrophic lateral sclerosis; $\mathbf{A U C}=$ area under the curve; $\mathbf{M S}=$ multiple sclerosis; $\mathbf{N M R}=$ nuclear magnetic resonance; PLS-DA = partial least squares discriminant analysis; $\mathbf{P P}=$ primary progressive; $\mathbf{R O C}=$ receiver operator characteristic; $\mathbf{R} \mathbf{R}=$ relapsing-remitting; $\mathbf{S P}=$ secondary progressive.

The transition from relapsing-remitting (RR) to secondary progressive (SP) multiple sclerosis (MS) occurs subtly and is difficult to define clinically. ${ }^{1}$ Identifying biomarkers that can distinguish between the different clinical phenotypes of MS is an important goal to ensure that the appropriate treatment regimens are adopted in a timely fashion. ${ }^{2-4}$ Furthermore, such biomarkers may provide new insight into the pathologic basis for the progressive process and lead to the development of effective treatments for disability prevention. ${ }^{5}$

Metabolomic profiling of biofluids with high-resolution proton nuclear magnetic resonance (NMR) spectroscopy and partial least squares discriminant analysis (PLS-DA), ${ }^{6}$ a multivariate statistical pattern recognition technique, can be used to identify metabolites that vary in a correlated fashion within individual groups. ${ }^{78}$ This approach enables patterns of metabolite variation that are characteristic of a specific disease to be defined, rather than requiring identification of a unique, candidate-led biomarker. ${ }^{9}$ We have previously used this technique to show that animals with either predominantly macrophage-rich or neutrophil-rich brain pathologies

\footnotetext{
*These authors contributed equally to this work and are listed alphabetically.

From the CR-UK/MRC Gray Institute for Radiation Oncology and Biology (A.M.D., J.R.L., N.R.S.), Department of Pharmacology (A.M.D., D.C.A.), Department of Chemistry (A.M.D., B.G.D., T.D.W.C.), Nuffield Department of Clinical Neurosciences (A.C., L.M., M.R.T.), and Nuffield Department of Medicine (G.K.W.), University of Oxford; and the Department of Biochemistry (J.L.G., J.P.), University of Cambridge, UK.

Go to Neurology.org for full disclosures. Funding information and disclosures deemed relevant by the authors, if any, are provided at the end of the article.
} 
can be differentiated from each other solely on the basis of their urine metabolite composition. ${ }^{10}$ Using a similar approach, characteristic metabolite patterns were identified in urine samples in a marmoset model of MS, as well as in samples from patients with MS compared with either healthy volunteers or other neurologic controls. ${ }^{11}$ These findings support the concept that biofluids analyzed by NMR/PLS-DA may be highly sensitive, not just to the presence of pathology within the brain, but to differences and changes in pathologic processes. In this study, we used NMR/PLS-DA analysis of serum metabolite composition to determine whether it is possible to objectively differentiate between MS clinical subtypes on the basis of metabolomic biofluid analysis.
METHODS Biofluid samples. We obtained serum samples from 3 independent cohorts (A, B, and C) of patients with MS (RR, SP, and primary progressive [PP]), as well as age- and sexmatched control volunteers, recruited from the John Radcliffe Hospital, Oxford, UK (see STARD flowchart of patient recruitment, exclusion, and methodology in figure 1). We diagnosed patients with MS using the gold standard 2001 and 2005 McDonald criteria, with at least 2 clinical attacks for RR phenotypes $^{12,13}$ and continuous worsening of neurologic impairment over at least 12 months, not explained by incomplete recovery from relapses, for those defined as SPMS. Of the 58 patients with SPMS in the study, 53 did not have a relapse in the 2 years before and after sampling, 3 had one relapse, and data on the remaining 2 are not available. No patients with PPMS had any recorded relapses. A consultant neurologist performed the clinical assessments of all patients with MS (J.P.). Overall patient demographics are shown in table 1, and a more detailed breakdown together with a list of therapies is provided in table e-1 on the Neurology ${ }^{\circledR}$ Web site at Neurology.org. Blood samples were collected and allowed to clot for 30 minutes at room temperature in Vacutainers containing clot-activator and gel (BD, $\mathrm{UK})$. Samples were centrifuged $\left(3,000 g_{\mathrm{n}}, 10\right.$ minutes $)$ and the serum aliquoted and stored at $-80^{\circ} \mathrm{C}$. Samples were excluded

Figure 1 STARD flowchart of multiple sclerosis patient recruitment and general methodology

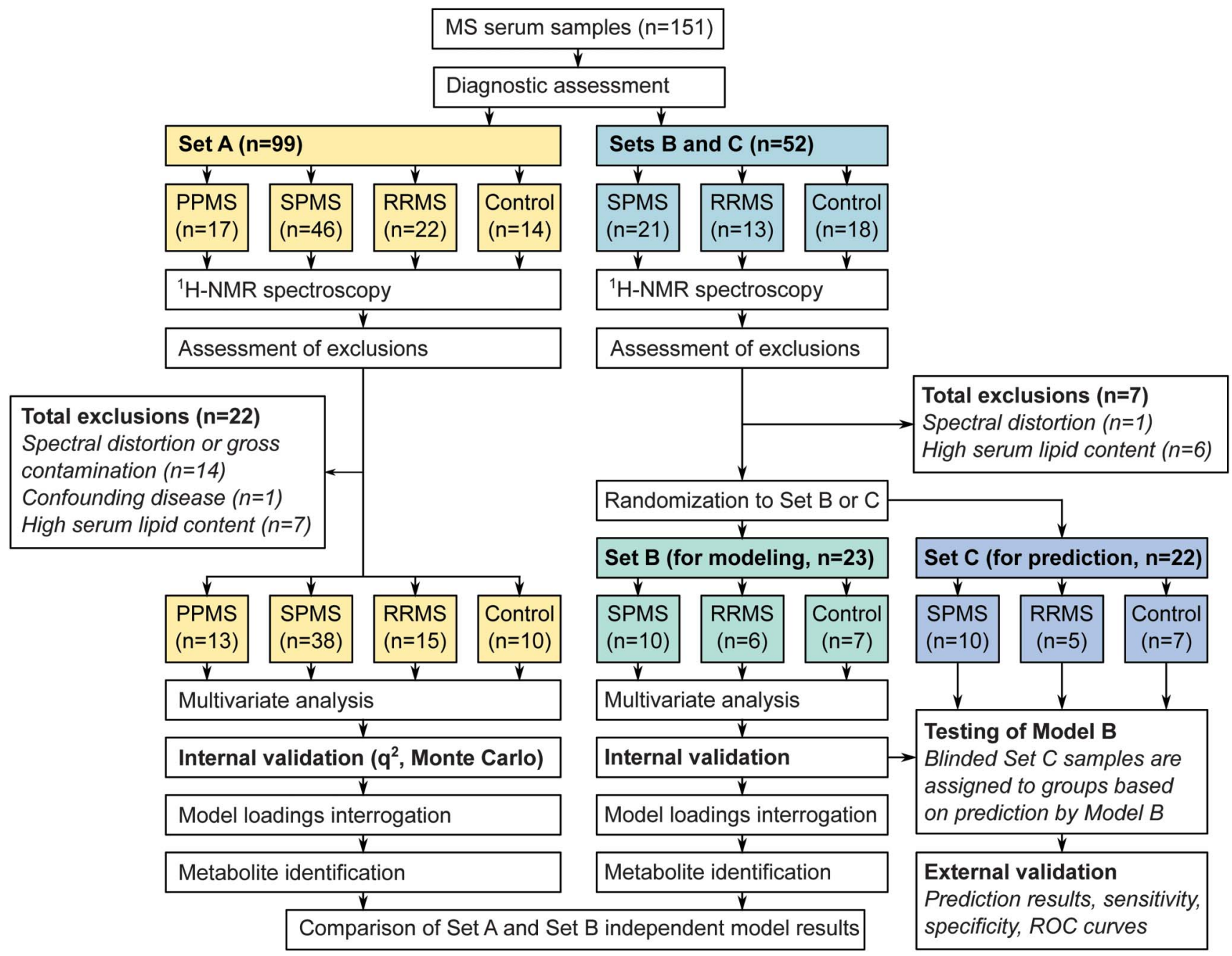

MS = multiple sclerosis; NMR = nuclear magnetic resonance; PPMS = primary progressive multiple sclerosis; ROC = receiver operator characteristic; RRMS = relapsing-remitting multiple sclerosis; SPMS = secondary progressive multiple sclerosis. 
Table 1 Patient demographic information

\begin{tabular}{lllll} 
& PPMS & RRMS & SPMS & \multicolumn{1}{c}{ Control } \\
\hline Median (range) age, y & $58(43-63)^{\mathrm{a}}$ & $40(22-62)$ & $51(28-65)^{\mathrm{b}}$ & $54(18-75)$ \\
Female, $\mathrm{n}(\%)$ & $6(46)$ & $15(58)$ & $43(74)$ & $16(67)$ \\
Male, $\mathrm{n}(\%)$ & $7(54)$ & $11(42)$ & $15(26)$ & $8(33)$ \\
Median (IQR) EDSS & $6(4.4-6.4)$ & $2(1-5.4)$ & $6(5.75-6.5)^{\mathrm{c}}$ & $0(0-0)^{\mathrm{d}}$ \\
Median (range) TSR, mo & $\mathrm{NA}$ & $20(1-161)$ & $\mathrm{NA}$ & $\mathrm{NA}$ \\
Median (range) DD, mo & $73(17-333)$ & $72(6-318)$ & $160(65-430)^{\mathrm{c}}$ & $0(0-0)^{\mathrm{d}}$
\end{tabular}

Abbreviations: DD = disease duration; EDSS = Expanded Disability Status Scale; IQR = interquartile range; NA = not applicable; PPMS = primary progressive multiple sclerosis; RRMS = relapsing-remitting multiple sclerosis; $\mathrm{SPMS}=$ secondary progressive multiple sclerosis; TSR = time since relapse.

More detailed breakdown of demographics and therapies received is given in table e-1.

a $p<0.001$ vs RRMS.

${ }^{\mathrm{b}} \mathrm{p}<0.05$ vs RRMS.

${ }^{\mathrm{c}} \mathrm{p}<0.001$ with respect to RRMS.

$d_{p}<0.001$ vs PPMS, RRMS, and SPMS.

from analysis if patients were found to have another major disease (e.g., diabetes mellitus $[n=1])$, if the spectral quality was poor or grossly distorted $(n=15)$, or if the prepared sample was found to have a lipid content greater than $2 \times$ SD from the mean of the group $(\mathrm{n}=13$, appendix e-1). For comparison to other neurologic diseases, serum was obtained from patients with either amyotrophic lateral sclerosis (ALS, $\mathrm{n}=20$, diagnosed by M.R.T.) or Alzheimer disease ( $\mathrm{AD}, \mathrm{n}=10$, diagnosed by G.K.W.) and compared with age-matched samples from healthy volunteers at the respective disease clinics ( $\mathrm{n}=14$ and 20 , respectively).

Standard protocol approvals, registrations, and patient consents. MS samples were collected and analyzed with ethical approval between 1995 and 2012 under project 08/H0604/ 155. ALS and AD patient samples were obtained from tissue banks and analyzed as part of the Oxford Study for Biomarkers in Motor Neurone Disease (BioMOx, approved by the South Oxfordshire Research Ethics Committee 08/H0605/85) or the OPTIMA and Challenge cohorts with prior approval from the local research ethics committee (COREC 1656 and 96.243, respectively).

NMR spectroscopy. A 1D proton NMR spectrum was obtained for each sample using a Carr-Purcell-Meiboom-Gill pulse sequence as previously described, ${ }^{14}$ with some slight modifications. Prior to spectroscopy, samples were defrosted at $4^{\circ} \mathrm{C}$, then further centrifuged $\left(100,000 \times g, 30\right.$ minutes, $\left.4^{\circ} \mathrm{C}\right)$. Serum $(150 \mu \mathrm{L})$ was mixed with $0.24 \mathrm{M}$ sodium phosphate NMR buffer prepared in $\mathrm{D}_{2} \mathrm{O}(\mathrm{pH}$ 7.4) to a final volume of $600 \mu \mathrm{L}$. Mixed samples were transferred to a $5-\mathrm{mm}$ NMR tube and spectra acquired using a $700-\mathrm{MHz}$ spectrometer operating at 16.4T (Bruker Avance III, Billerica, MA). One sample from each batch was analyzed by $2 \mathrm{D}$ spectroscopy to aid metabolite identification. More detailed NMR methods are provided in appendix e-1.

Data preprocessing. Spectra were imported into MATLAB (Mathworks, Natick, MA) and scaled using the internal standard to ensure correct amplitude. Further preprocessing corrected for subtle shifts in peak position arising from $\mathrm{pH}$ differences between samples. ${ }^{15}$ The remaining processing was carried out as previously described,${ }^{14}$ with slight modifications. Briefly, the spectra were subdivided into 0.02 -ppm buckets from 0.20 to $9.60 \mathrm{ppm}$ ( $\delta=$ midpoint of integral region). In each spectrum, buckets in the
4.30-5.00 ppm region, which is highly variable owing to imperfect water suppression, were excluded. In addition, buckets containing gross contaminants (e.g., organic solvent) were excluded from analysis. Each remaining bucket was integrated, yielding 420 independent variables for each sample. Prior to multivariate statistical analysis, data were scaled using the Pareto variance to suppress noise. More detailed methodology is available in appendix e-1.

Multivariate statistical analysis. For each comparison, we used SIMCA 13 (Umetrics, Sweden) to construct a PLS-DA model that best explained the differences between the variables for the groups being studied. Each model was assessed for predictivity by calculating the $\mathrm{q}^{2}$ value. $\mathrm{A} \mathrm{q}^{2}>0$ is predictive and it is generally accepted that $\mathrm{q}^{2}>0.4$ is the threshold for significance in biological modeling. ${ }^{14}$ Models were further validated by a Monte Carlo method where 100 models were built using random group assignments. Only models where the genuine $\mathrm{q}^{2}$ was higher than $95 \%$ of the randomly generated $\mathrm{q}^{2}$ values were considered significant. More detailed statistical methods are included in appendix e-1.

Buckets driving separations were identified using variable importance and contribution plots for each model. Metabolites within buckets were identified using 2D spectroscopy, literature values, and reference to the human metabolome database. ${ }^{16-18}$ To confirm identity of singlet peaks, predicted metabolites were spiked into samples as authentic references.

Independent validation. Two sets of patients, A and B, were used to build independent predictive models. Each model's loadings and contributions were compared to ensure similarity in variables driving separation. In addition, a third independent set of samples, C, was used as a testing set. A researcher blinded to all clinical information tested the ability of the Set B model to predict group membership of the unknown Set $\mathrm{C}$ samples. The results were expressed as a $2 \times 2$ contingency table and receiver operator characteristic (ROC) curves were constructed. Model membership probability thresholds derived from the modelbuilding set were used to determine sensitivity and specificity in the prediction set (Set C).

RESULTS Initial MS sample set (Set A). Spectra were obtained from the Set A serum samples and processed as described above to give integral values for each 
independent bucket. Example spectra from a patient with RRMS and from a patient with SPMS are shown in figure e-1. Models built with data from Set A serum samples showed a significant separation between RR and SP groups $\left(\mathrm{q}^{2}=0.45\right.$, figure $\left.2 \mathrm{~A}\right)$. Examination of the loadings revealed that fatty acids $\left(\delta_{\mathrm{x}-\mathrm{y}}=0.88,1.30,5.35\right)$, phosphocholine $\left(\delta_{\mathrm{x}-\mathrm{y}}=\right.$ $3.23)$, an $N$-acetyl species $\left(\delta_{\mathrm{x}-\mathrm{y}}=2.03\right)$, and glucose

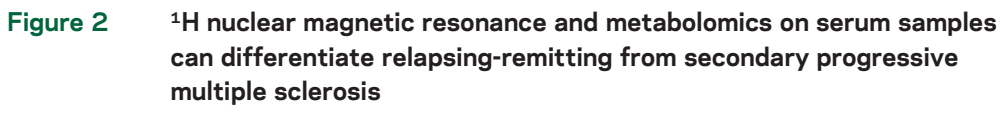

Figure $2 \quad{ }^{1} \mathrm{H}$ nuclear magnetic resonance and metabolomics on serum samples can differentiate relapsing-remitting from secondary progressive multiple sclerosis

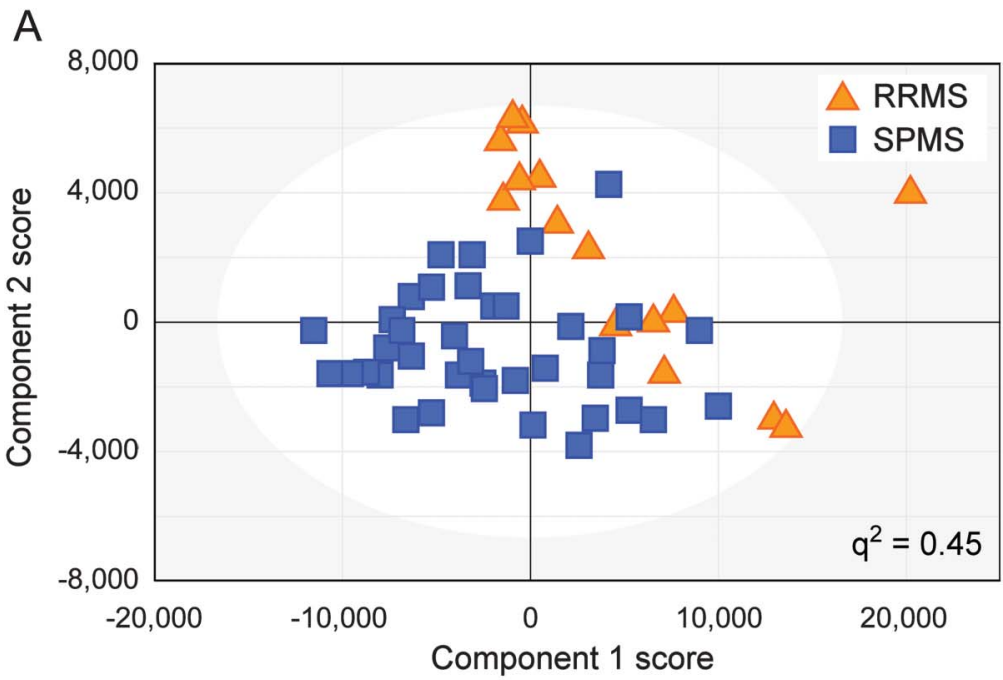

B

\begin{tabular}{|c|c|c|c|c|}
\hline Serum & RRMS & PPMS & SPMS & \\
\hline \multirow{3}{*}{$\begin{array}{l}\overline{\text { o }} \\
\text { 듬 } \\
\text { O }\end{array}$} & 0.61 & n.p. & 0.45 & RRMS \\
\hline & & 0.70 & n.p. & PPMS \\
\hline & & & 0.42 & SPMS \\
\hline
\end{tabular}

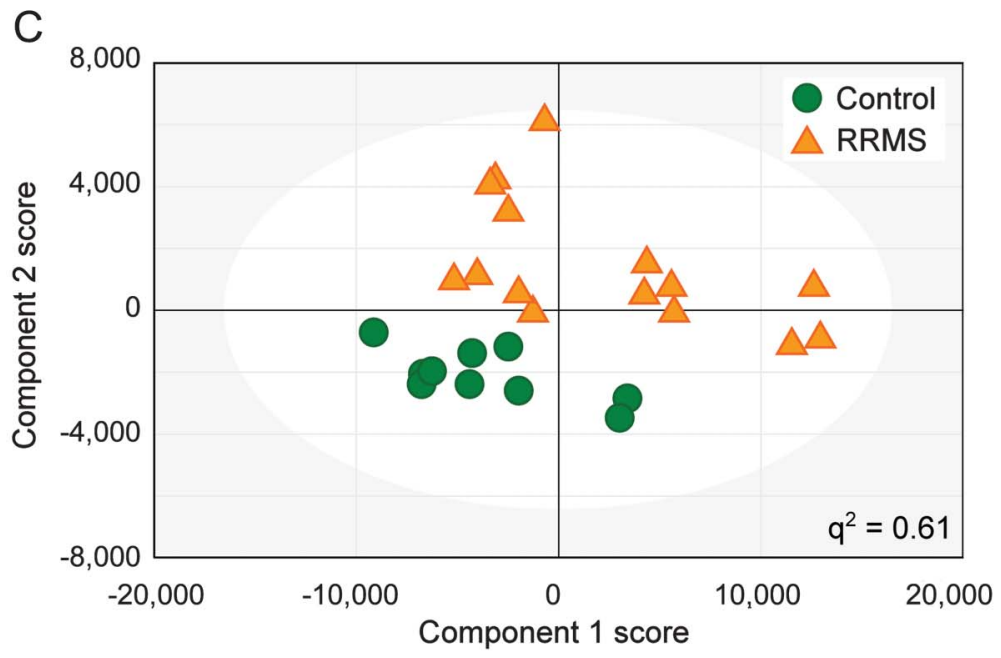

Serum analysis: (A) Partial least squares discriminant analysis plot of serum samples comparing patients with relapsing-remitting multiple sclerosis (RRMS) (orange triangles) and patients with secondary progressive multiple sclerosis (SPMS) (blue squares). (B) Table to show the $\mathrm{q}^{2}$ values of the models from Set $A$ serum samples. n.p. $=$ not predictive (i.e., $q^{2}<0$ ). (C) Patients with RRMS (orange diamonds) and control volunteers (green circles). PPMS = primary progressive multiple sclerosis. $\left(\delta_{x-y}=3.25,3.75,3.91\right)$ were decreased in SPMS with respect to RRMS, whereas other fatty acids and $\beta$-hydroxybutyrate $\left(\delta_{x-y}=1.19\right)$ were increased. For other intra-MS comparisons, the model comparing the PP and RR groups appeared to differentiate 2 clear groups (figure e-2). However, a small number of overlapping patients in each group resulted in a nonpredictive $\mathrm{q}^{2}$ value $(-0.11)$. The $\mathrm{PP}$ vs $\mathrm{SP}$ model also returned a nonpredictive $\mathrm{q}^{2}$ value $(-0.02)$, with marked overlap (figure e-2).

We subsequently investigated the separation of patients with MS from an age- and sex-matched control cohort. A model differentiating all patients with MS from the control cohort was significantly predictive $\left(\mathrm{q}^{2}=0.41\right)$. Furthermore, the comparisons between individual stages of MS and control groups were also significant (figure 2, B and C). All models were successfully validated using the cross-validation method described above. Interestingly, when comparing each of the individual stages of MS against the controls, 2 metabolites were consistent across the groups: glucose $\left(\delta_{x-y}=3.25,3.75,3.91\right)$ and phosphocholine $\left(\delta_{x-y}=\right.$ 3.23) were both reduced. Further examination of both the RR vs control and SP vs control model loadings showed lactate $\left(\delta_{\mathrm{x}-\mathrm{y}}=1.32\right)$, a broad singlet-like resonance tentatively assigned to $N$-acetyl species $\left(\delta_{\mathrm{x}-\mathrm{y}}=2.03\right.$, previously assigned as $N$-acetyl glycoproteins $s^{10}$ ), and some fatty acids were decreased in the patients with RRMS or patients with SPMS relative to controls. A separate subset of fatty acids was found to be elevated in the patients with RRMS and patients with SPMS relative to controls. Examination of the PP vs control model loadings showed that, in contrast to RRMS and SPMS, lactate, $\mathrm{N}$-acetyl species, and some fatty acids were increased in patients with PPMS relative to controls. Detailed metabolite findings are presented in table e- 2 .

Independent MS sample set analysis (Sets B and C). Samples from independent Set B were used in the same manner as the samples from Set $A$ and 3 new models were generated: Control $_{B}$ vs $\mathrm{RRMS}_{\mathrm{B}}$, Control ${ }_{\mathrm{B}}$ vs $\mathrm{SPMS}_{\mathrm{B}}$, and $\mathrm{RRMS}_{\mathrm{B}}$ vs $\mathrm{SPMS}_{\mathrm{B}}$. In each case, the models from Set $\mathrm{B}$ were significantly predictive $\left(q^{2}>0.4\right)$ and were all validated in the same manner as the models from Set A. $\left(\right.$ Control $_{\mathrm{B}}$ vs $\mathrm{RRMS}_{\mathrm{B}}, \mathrm{q}^{2}=$ 0.62; Control $_{B}$ vs $S_{P M S}, q^{2}=0.77$; and $R_{R M S}$ vs $\mathrm{SPMS}_{\mathrm{B}}, \mathrm{q}^{2}=0.48$.) Moreover, upon examination of the loadings, the same metabolites were found to be responsible for the separations observed between groups in this secondary dataset with the exception of glucose, which had a lower variable importance in separating patients with SPMS from patients with RRMS in Set B.

Each new model generated from Set B was tested for its predictive ability using Set C, the independent 
dataset that had not been used in building models. All Set B models were both sensitive and specific at predicting group membership for patients: $R_{R M S_{B}}$ vs $\mathrm{SPMS}_{\mathrm{B}}$, sensitivity $=0.9$ and specificity $=0.8$; $\mathrm{RRMS}_{\mathrm{B}}$ or $\mathrm{SPMS}_{\mathrm{B}}$ vs Control $_{\mathrm{B}}$, sensitivity and specificity $=1.0$. Graphical representations of models and prediction results (figure $3, \mathrm{~A}-\mathrm{C}$ ), and contingency tables (figure 3, D-F), demonstrate sensitivity and specificity of the models (Fisher exact test, 2-tailed). ROC plots (figure $3 \mathrm{G}$ ) yielded areas under the curve (AUC) significantly greater than 0.5 , indicating good predictive power (control vs RRMS, AUC $=1.00$, $p=0.0045$; control vs SPMS, AUC $=1.00, p=$ 0.0006 ; SPMS vs RRMS, AUC $=0.94, p=0.0071)$.

Figure 3 Partial least squares discriminant analysis models and receiver operator characteristic curves demonstrate the selectivity and specificity of the model separating relapsing-remitting and secondary progressive multiple sclerosis

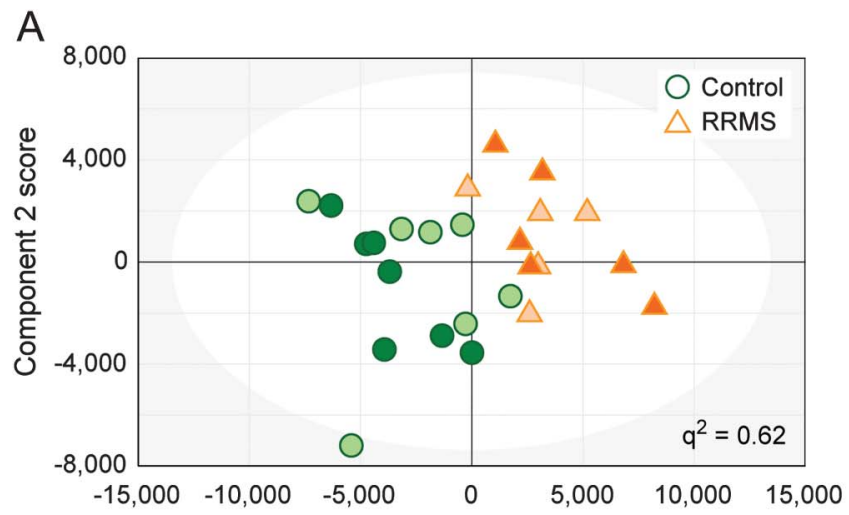

D

\begin{tabular}{ccc}
\hline \multirow{2}{*}{$\begin{array}{c}\text { True } \\
\text { membership }\end{array}$} & \multicolumn{2}{c}{ Predicted membership } \\
\cline { 2 - 3 } & Control & RRMS \\
\hline Control & 0 & 7 \\
\hline RRMS & 0 & 5 \\
\hline
\end{tabular}

$\mathrm{E}$

\begin{tabular}{ccc}
\hline \multirow{2}{*}{$\begin{array}{c}\text { True } \\
\text { membership }\end{array}$} & \multicolumn{2}{c}{ Predicted membership } \\
\cline { 2 - 3 } & Control & SPMS \\
\hline Control & 0 & 7 \\
\hline SPMS & 0 & 10 \\
\hline
\end{tabular}

B

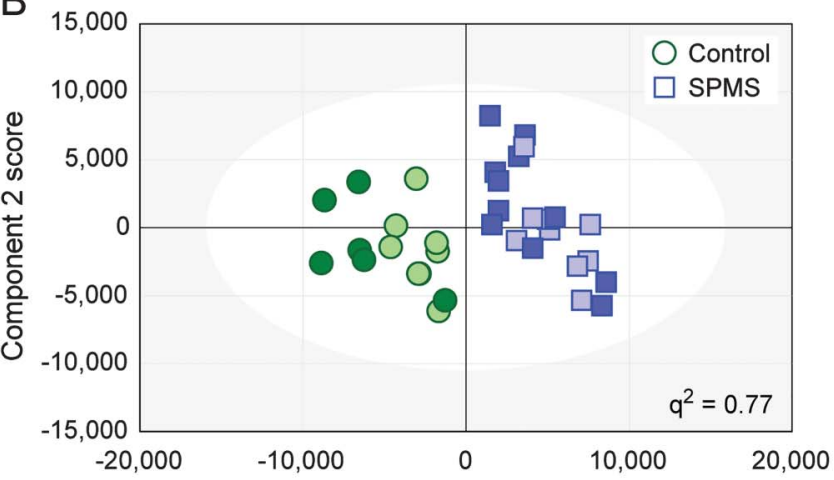

C
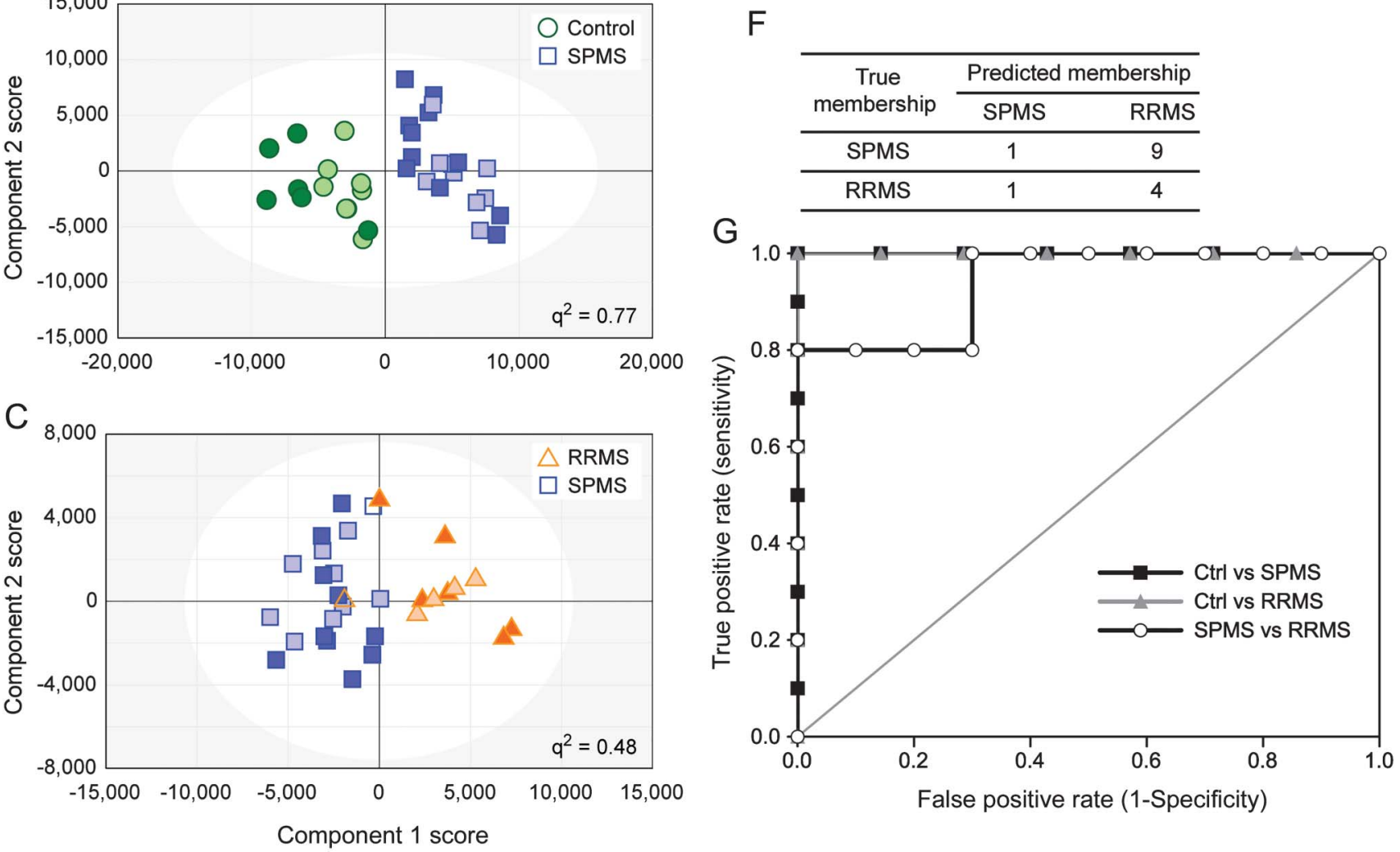

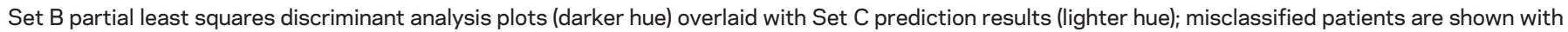

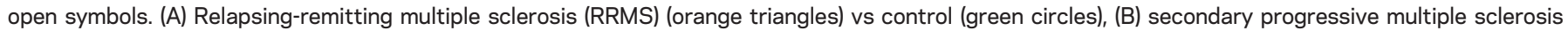

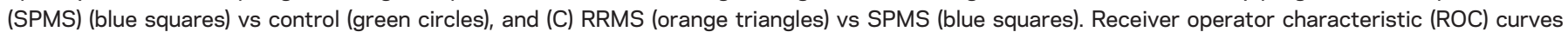

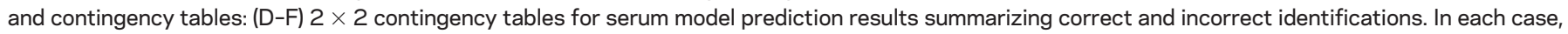

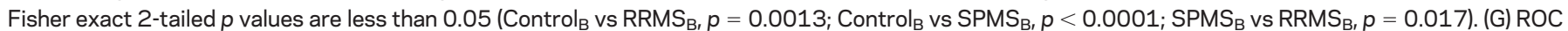

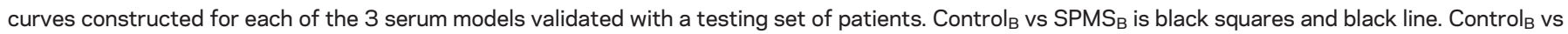

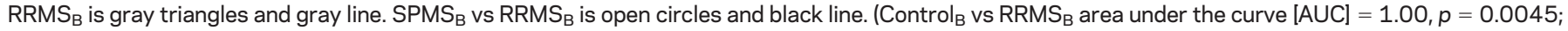
Control $_{B}$ vs SPMS $A U C=1.00, p<0.0001 ;$ SPMS $_{B}$ vs RRMS $_{B}$ AUC $=0.94, p=0.007$.) 
Comparison with other neurologic diseases. The control vs RRMS model (figure 2C) was compared with models separating ALS and AD from age-matched control samples (figure $4, \mathrm{~A}$ and $\mathrm{B}$, respectively). Neither of the latter 2 models was predictive, with a strong overlap of groups in both cases.

Effect of therapeutic agents, age, and sex. No significant models could be generated when modeling patients within our study randomized to either active therapy or placebo arms of the Cupid trial (tetrahydrocannabinol, $\mathrm{q}^{2}=-0.08$ ) or BioMS trial (Dirucotide, MBP$\left.8298, \mathrm{q}^{2}=-0.16\right)$. Thus, the presence of these therapeutic agents did not contribute to the separations observed. Full details of therapies received are given in table e-1. Furthermore, when considering age as a factor within the control population, a model between older $(\geq 46)$ and

Figure 4 Models comparing different neurologic diseases to their respective controls

A

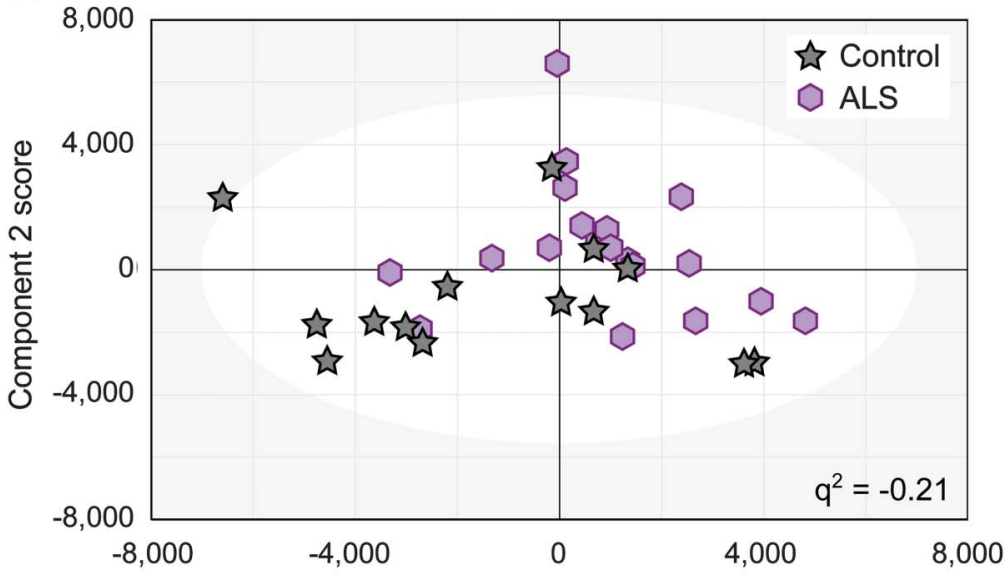

B

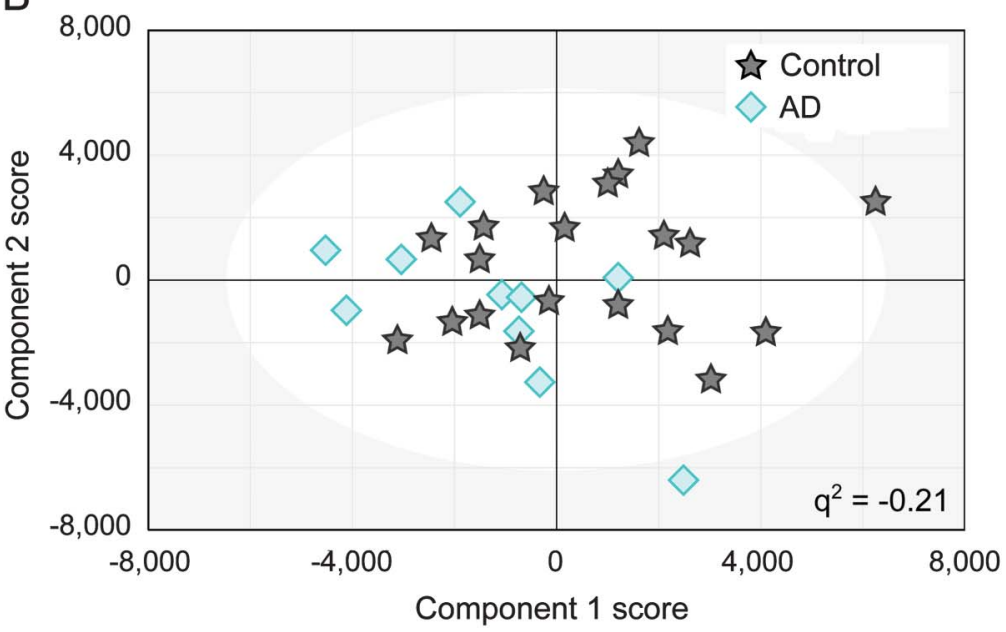

Models to probe separation between 2 different neurologic diseases and control volunteers from their respective disease clinics. (A) Patients with amyotrophic lateral sclerosis (ALS) (purple hexagons) and control volunteers (gray stars); model is nonpredictive $\left(\mathrm{q}^{2}=-0.21\right.$ ). (B) Patients with Alzheimer disease (AD) (turquoise diamonds) and control volunteers (gray stars); model is nonpredictive $\left(q^{2}=-0.21\right)$. younger volunteers $(<46)$ was not predictive $\left(\mathrm{q}^{2}=\right.$ -0.19 ). The cutoff of 46 was chosen as it lay halfway between the mean ages of the RRMS and SPMS cohorts. Likewise, when the sex of patients was highlighted on existing models (e.g., the Set A SPMS vs RRMS model), no discernible patterns were evident, with both sexes being distributed evenly. Thus, none of these variables (presence of therapeutic agent, age, or sex) contributed to the significant separations observed in our MS models.

DISCUSSION Using PLS-DA metabolomics analysis of serum NMR spectra, it was possible to generate validated predictive models that distinguished RRMS from SPMS, as well as separating each of the MS patient groups (RRMS, SPMS, or PPMS) from healthy control cohorts. This is Class II evidence. The majority of serum metabolites that underlay the model separations were identified. The sensitivity of the models to specific metabolites varied between each of the MS groups when compared with the control cohort, and most notably between PPMS and either of the other 2 groups, indicating sensitivity to the different disease states. The findings in other diseases (ALS and AD) served as a negative control, and confirmed that the predictive models generated in the MS cohorts were based on disease-specific metabolite changes and did not reflect common neurodegenerative processes per se.

It is of particular interest that our serum models generated a positive and significant separation between RR and SP patient groups. This separation was highly robust, with 2 independent sample sets (A and $\mathrm{B}$ ) producing equivalent predictive models and identifying the same underlying metabolites. The only difference in the metabolites identified was glucose, which had a lesser influence in driving the models derived from Set B than Set A. This difference is likely to have arisen from variations in the timing of sample collection in the clinic and the period after the last meal. Critically, models created from Set B accurately predicted group membership of samples from the third, independent set (Set C). These findings strongly support the concept that NMR/PLS-DA analysis of serum samples can reliably differentiate between the RR and SP stages of MS disease progression. Based on the positive outcome of these relatively small, cross-sectional studies, further larger cohort, multicenter longitudinal trials are warranted to assess the potential of this approach both as a means to monitor transitioning from RRMS to SPMS and as a surrogate marker of time to SP disease in treatment trials.

Our models were not able to separate the progressive groups of MS from each other. This supports the 
view that a similar pathologic mechanism underlies these phases. ${ }^{19}$ However, the lack of a predictive model between the PP and RR patients appeared to be disrupted by just 4 patients ( $3 \mathrm{RR}$ and $1 \mathrm{PP}$ ) lying in the incorrect model region, suggesting that the underlying pathologic mechanisms of RRMS and PPMS are distinct. Although it is easy to distinguish RRMS from PPMS in the clinical setting, understanding the underlying pathogenic basis for PP disease is important. Thus, these observations warrant investigation and validation in larger cohorts. Further, a study to assess the potential of this approach in predicting conversion of clinically isolated syndromes to RRMS would be valuable and might help identify those patients for whom early treatment would be beneficial.

Previous studies of biomarkers in neurologic diseases, including MS, have investigated either individual or small numbers of indicators. ${ }^{20-23}$ However, despite differences identified at a group level, predictive value for an individual has been low. ${ }^{24}$ Whereas it is possible to separate patients with MS from controls using techniques such as MRI, ${ }^{12,13,25}$ or using a metabolomics-based approach on CSF samples, ${ }^{26}$ to our knowledge no studies have been reported in which patients with RRMS and SPMS have been separated from each other on the basis of serum NMR. As an anecdotal example of the potential strength of this approach, one patient with RRMS was found persistently to lie in the SPMS portion of the model. As this patient had been categorized some years previously, her neurologist was consulted to obtain her current status. The sample was collected in 2008 and at follow-up 1 year later the patient reported progressing over that year. By 2010, there was no doubt that the patient was progressing. These findings suggest that the metabolomic profile of the patient's serum in 2008 was an early indicator of entering the progressive stage of disease.

It is important to note that, owing to its classbased learning approach, PLS-DA analysis reduces the impact of underlying conditions such as therapeutic status or diet-based effects, since these are likely to be randomly distributed throughout our sample population. If any variance in a population does not explain the difference between the user-defined classes, it is considered less important. This was demonstrated in our data as some patients were on blinded clinical trials. The models could discriminate between individuals with RRMS or SPMS in a manner that was independent of treatment regimen, suggesting that the metabolite changes caused by the disease progression were more consistent than those associated with the metabolism of any drugs present. This advantage of the PLS-DA approach is essential if it is to be useful clinically, as it must be able to discriminate between people with the specific disease states despite these nonspecific population variations.

The PLS-DA models developed here could be used to mark the change from RRMS to SPMS in clinical studies and, thus, enable onset of secondary progression to be used as an outcome measure in therapeutic trials. ${ }^{27}$ This is likely to be a more important outcome than relapse activity and better than shortterm (3-6 months) disability change, which includes relapse-associated disability as well as progression. Moreover, this approach may identify key metabolites involved in the pathogenesis underlying progression of $\mathrm{MS}^{28}$ and identify targets for the development of novel therapies.

\section{AUTHOR CONTRIBUTIONS}

A.M. Dickens: contributed to the design of the principal experiments, performed the sample preprocessing for the NMR experiments, assisted with running the NMR experiments, performed the multivariate analysis, contributed to writing and proofing of the manuscript. J.R. Larkin: contributed to the design of the principal experiments, performed the sample preprocessing for the NMR experiments, assisted with running the NMR experiments, performed the multivariate analysis, contributed to writing and proofing of the manuscript. J.L. Griffin: designed the NMR sequences used in this study and assisted with the multivariate analysis, contributed to writing and proofing of the manuscript. A. Cavey: collected the MS samples used in this study and clinical data required for the samples, contributed to writing and proofing of the manuscript. L. Matthews: collected the MS samples used in this study and clinical data required for the samples, contributed to writing and proofing of the manuscript. M.R. Turner: collected the ALS samples used in this study, contributed to writing and proofing of the manuscript. G.K. Wilcock: collected the Alzheimer samples used in this study, contributed to writing and proofing of the manuscript. B.G. Davis: assisted with the interpretation of the NMR spectra and multivariate analysis, contributed to writing and proofing of the manuscript. T.D.W. Claridge: ran the NMR experiments in this study and assisted with metabolite identification from the NMR spectra, contributed to writing and proofing of the manuscript. J. Palace: contributed to the design of the principal experiments, provided the samples from the patients with MS and aided interpretation of the results, contributed to writing and proofing of the manuscript. D.C. Anthony: contributed to the design of the principal experiments and interpretation of the results, contributed to writing and proofing of the manuscript. N.R. Sibson: contributed to the design of the principal experiments, supervised all work and helped with the analysis and interpretation of the results, contributed to writing and proofing of the manuscript.

\section{STUDY FUNDING}

Supported by the Medical Research Council: research grant G0401438 (N.R.S.), G0901996 (L.M.), and Capacity Building Studentship to fund AD (N.R.S., D.C.A., B.G.D.). N.R.S. and J.R.L. were partly funded by Cancer Research UK (grant C5255/A12678). B.G.D. is a Royal Society Wolfson Research Merit Award recipient. G.K.W. was partly funded by the NIHR Biomedical Research Centre Program, Oxford.

\section{DISCLOSURE}

A. Dickens was funded by the Medical Research Council, UK, under a Capacity Building Studentship. J. Larkin was partly funded by Cancer Research, UK, grant C5255/A12678. J. Griffin and A. Cavey report no disclosures. L. Matthews is funded by the Medical Research Council, UK, grant G0901996. M. Turner reports no disclosures. G. Wilcock is partly funded by the NIHR Biomedical Research Centre Program, Oxford. B. Davis is a Royal Society Wolfson Research Merit Award and received research support from the Medical Research Council, UK, in the form of a Capacity Building Studentship (to fund AD). T. Claridge and J. Palace report no disclosures. D. Anthony received funding 
from the Medical Research Council, UK, in the form of a Capacity Building Studentship (to fund AD). N. Sibson received partial funding for this work from the Medical Research Council, UK, under grant G0401438 and a Capacity Building Studentship (to fund AD). In addition, Dr. Sibson was partly funded by Cancer Research, UK, grant C5255/A12678. Go to Neurology.org for full disclosures.

Received September 20, 2013. Accepted in final form June 4, 2014.

\section{REFERENCES}

1. Jia Y, Wu T, Jelinek CA, et al. Development of protein biomarkers in cerebrospinal fluid for secondary progressive multiple sclerosis using selected reaction monitoring mass spectrometry (SRM-MS). Clin Proteomics 2012;9:9.

2. Menge T, Weber MS, Hemmer B, et al. Disease-modifying agents for multiple sclerosis: recent advances and future prospects. Drugs 2008;68:2445-2468.

3. Rieckmann P, Smith KJ. Multiple sclerosis: more than inflammation and demyelination. Trends Neurosci 2001; 24:435-437.

4. Jones JL, Coles AJ. New treatment strategies in multiple sclerosis. Exp Neurol 2010;225:34-39.

5. Hauser SL, Chan JR, Oksenberg JR. Multiple sclerosis: prospects and promise. Ann Neurol 2013;74:317-327.

6. Holmes E, Foxall PJ, Nicholson JK, et al. Automatic data reduction and pattern recognition methods for analysis of ${ }^{1} \mathrm{H}$ nuclear magnetic resonance spectra of human urine from normal and pathological states. Anal Biochem 1994;220:284-296.

7. Lindon J, Holmes E, Nicholson J. Metabonomics in pharmaceutical R \& D. FEBS J 2007;274:1140-1151.

8. Salek RM, Xia J, Innes A, et al. A metabolomic study of the CRND8 transgenic mouse model of Alzheimer's disease. Neurochem Int 2010;56:937-947.

9. Lindon JC, Holmes E, Nicholson JK. Pattern recognition methods and applications in biomedical magnetic resonance. Prog Nucl Magn Reson Spectrosc 2001;39:1-40.

10. Griffin JL, Anthony DC, Campbell SJ, et al. Study of cytokine induced neuropathology by high resolution proton NMR spectroscopy of rat urine. FEBS Lett 2004;568: 49-54.

11. 't Hart BA, Vogels JT, Spijksma G, Brok HP, Polman C, van der Greef J. H-1-NMR spectroscopy combined with pattern recognition analysis reveals characteristic chemical patterns in urines of MS patients and non-human primates with MS-like disease. J Neurol Sci 2003;212:21-30.

12. McDonald W, Compston A, Edan G, et al. Recommended diagnostic criteria for multiple sclerosis: guidelines from the International Panel on the diagnosis of multiple sclerosis. Ann Neurol 2001;50:121-127.

13. Polman CH, Reingold SC, Edan G, et al. Diagnostic criteria for multiple sclerosis: 2005 revisions to the "McDonald Criteria”. Ann Neurol 2005;58:840-846.
14. Waterman C, Currie R, Cottrell L, et al. An integrated functional genomic study of acute phenobarbital exposure in the rat. BMC Genomics 2010;11:9.

15. Tomasi G, van den Berg F, Andersson C. Correlation optimized warping and dynamic time warping as preprocessing methods for chromatographic data. J Chemom 2004;18:231-241.

16. Fan WMT. Metabolite profiling by one- and twodimensional NMR analysis of complex mixtures. Prog Nucl Magn Reson Spectrosc 1996;28:161-219.

17. Wishart DS, Tzur D, Knox C, et al. HMDB: the human metabolome database. Nucleic Acids Res 2007; 35:D521-D526.

18. Wishart DS, Knox C, Guo AC, et al. HMDB: a knowledgebase for the human metabolome. Nucleic Acids Res 2009;37:D603.

19. Scalfari A, Neuhaus A, Degenhardt A, et al. The natural history of multiple sclerosis, a geographically based study 10: relapses and long-term disability. Brain 2010;133: 1914-1929.

20. Whitaker JN, Kachelhofer RD, Bradley EL, et al. Urinary myelin basic protein-like material as a correlate of the progression of multiple sclerosis. Ann Neurol 1995;38: 625-632.

21. Whitaker JN, McKeehan A, Freeman DW. Monoclonal and polyclonal antibody responses to the myelin basic protein epitope present in human urine. J Neuroimmunol 1994;52:53-60.

22. Ott M, Demisch L, Engelhardt W, Fischer PA. Interleukin-2, soluble interleukin-2-receptor, neopterin, L-tryptophan and beta 2-microglobulin levels in CSF and serum of patients with relapsing remitting or chronic progressive multiple sclerosis. J Neurol 1993;241:108-114.

23. Giovannoni G, Lai M, Kidd D, et al. Daily urinary neopterin excretion as an immunological marker of disease activity in multiple sclerosis. Brain 1997;120:1-13.

24. Kuhle J, Leppert D, Petzold A, et al. Neurofilament heavy chain in CSF correlates with relapses and disability in multiple sclerosis. Neurology 2011;76:1206-1213.

25. Polman CH, Reingold SC, Banwell B, et al. Diagnostic criteria for multiple sclerosis: 2010 Revisions to the McDonald criteria. Ann Neurol 2011;69:292-302.

26. Tumani H, Hartung H-P, Hemmer B, et al. Cerebrospinal fluid biomarkers in multiple sclerosis. Neurobiol Disease 2009;35:117-127.

27. Lesko LJ, Atkinson A Jr. Use of biomarkers and surrogate endpoints in drug development and regulatory decision making: criteria, validation, strategies 1 . Annu Rev Pharmacol Toxicol 2001;41:347-366.

28. Frank R, Hargreaves R. Clinical biomarkers in drug discovery and development. Nat Rev Drug Discov 2003;2: 566-580. 


\section{Neurology}

\section{A type 2 biomarker separates relapsing-remitting from secondary progressive multiple sclerosis}

Alex M. Dickens, James R. Larkin, Julian L. Griffin, et al.

Neurology 2014;83;1492-1499 Published Online before print September 24, 2014

DOI 10.1212/WNL.0000000000000905

This information is current as of September 24, 2014

\section{Updated Information \&} Services

Supplementary Material

\section{References}

Citations

Subspecialty Collections

Permissions \& Licensing

Reprints including high resolution figures, can be found at: http://n.neurology.org/content/83/17/1492.full

Supplementary material can be found at: http://n.neurology.org/content/suppl/2014/09/24/WNL.0000000000000 905.DC2

http://n.neurology.org/content/suppl/2014/09/24/WNL.0000000000000 905.DC1

This article cites 28 articles, 1 of which you can access for free at: http://n.neurology.org/content/83/17/1492.full\#ref-list-1

This article has been cited by 2 HighWire-hosted articles: http://n.neurology.org/content/83/17/1492.full\#\#otherarticles

This article, along with others on similar topics, appears in the following collection(s):

Diagnostic test assessment

http://n.neurology.org/cgi/collection/diagnostic_test_assessment_ MRS

http://n.neurology.org/cgi/collection/mrs

Multiple sclerosis

http://n.neurology.org/cgi/collection/multiple_sclerosis

Information about reproducing this article in parts (figures,tables) or in its entirety can be found online at:

http://www.neurology.org/about/about_the_journal\#permissions

Information about ordering reprints can be found online:

http://n.neurology.org/subscribers/advertise

Neurology ${ }^{\circledR}$ is the official journal of the American Academy of Neurology. Published continuously since 1951, it is now a weekly with 48 issues per year. Copyright @ 2014 American Academy of Neurology. All rights reserved. Print ISSN: 0028-3878. Online ISSN: 1526-632X.

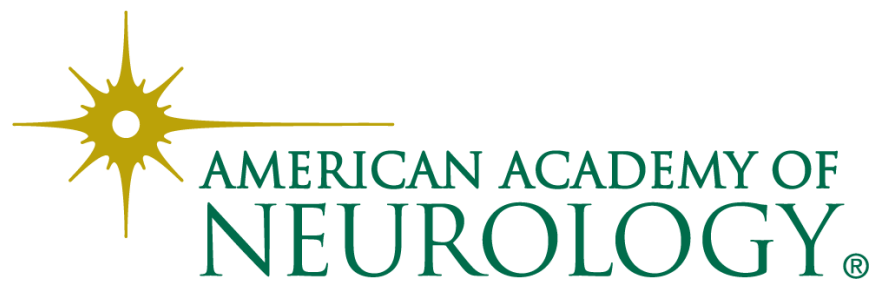

